\title{
Calidad de los registros en programa de detección oportuna de cáncer cervicouterino
}

\author{
Sandra Olimpia Gutiérrez-Enríquez* \\ Yolanda Terán-Figueroa** \\ Lucía Monreal-Delgado*** \\ Rafael Nieva-de-Jesús**** \\ Darío Gaytán-Hernández ${ }^{* * * * *}$
}

\begin{abstract}
*Enfermera. Maestra en Epidemiología y Administración en Salud. Doctora en Ciencias de la Salud Pública. Profesor Investigador de la Facultad de Enfermería y Nutrición. Universidad Autónoma de San Luis Potosí. San Luis Potosí. México.

**Enfermera. Maestra en Ciencias en Biología. Doctora en Ciencias en Biología. Profesor-Investigador de la Facultad de Enfermería y Nutrición. Universidad Autónoma de San Luis Potosí. San Luis Potosí. México.

***Enfermera. Maestra en Administración de la Atención de Enfermería. Especialista en Administración de la Unidad Médica de Alta Especialidad Nº48 del Instituto Mexicano del Seguro Social. San Luis Potosí. México.

****Médico Cirujano. Especialista en Medicina Familiar. Coordinador Clínico de Educación e Investigación del Hospital General de Zona №6 del Instituto Mexicano del Seguro Social. San Luis Potosí. México.

*****Ingeniero en Sistemas Computacionales. Maestro en Planeación y Sistemas. Doctor en Ciencias Ambientales. Profesor Investigador de la Facultad de Enfermería y Nutrición. San Luis Potosí. México.

Correspondencia: Dra. Yolanda Terán Figueroa. Dirección: Av. Niño Artillero 130, Zona Universitaria Poniente, C.P. 78240, San Luis Potosí, S.L.P., México. Teléfono: +52 (444) 8262300 Ext 5053. Correo electrónico: yolandat@uaslp.mx
\end{abstract}

\section{RESUMEN}

Introducción: el cáncer cervicouterino es un problema de salud pública en México, en 2012 se estimaron 528 ooo nuevos casos. Una línea de investigación con relación al programa de cáncer cervicouterino, es la calidad de los registros en cuanto a su legibilidad, completitud y precisión. Objetivo: evaluar la calidad de los registros realizados por el personal de salud en el servicio de Detección de Cáncer Cervicouterino. Materiales y Métodos: estudio transversal, realizado en el Instituto Mexicano del Seguro Social de San Luis Potosí, México, entre agosto de 2012 a septiembre de 2013. Ante un universo de 4139 registros, hoja de solicitud y resultados de la citología cervical, realizados durante enero a noviembre de 2012, se aplicó un muestreo probabilístico estratificado (1115 registros). Se utilizó una lista de verificación con 32 ítems divididos en siete apartados. Para analizar los datos se utilizaron tres indicadores: precisión, legibilidad y completitud. Se estableció una escala ordinal con el puntaje obtenido: bajo (0-64), medio (65-128) y alto (129-192). Se aplicó la prueba t de student para comparar los puntajes en la calidad de los registros. Resultados: en legibilidad se obtuvo un nivel medio en la calidad de los registros (80,6\%), en la completitud un 59,6\% en el nivel alto y en la precisión un 40,4\% en el nivel medio. El personal de enfermería obtuvo mejor calificación en la calidad de los registros que los cito-tecnólogos $(p=0,05)$. Conclusión: La calidad de los registros realizados por el personal de salud es media. MÉD.UIS. 2017;30(3):59-65.

Palabras clave: Cáncer Cervical. Garantía de la Calidad de Atención de Salud. Prueba de Papanicolaou.

\section{Quality of records in a timely screening program for cervical cancer}

\section{ABSTRACT}

Introduction: cervical cancer is a public health problem in Mexico, in 2012528000 new cases. One line of research related to the cervical cancer program is the quality of records in terms of readability, completeness, and accuracy. Objective: to evaluate the quality of the records made by the health personnel in the Cervical Cancer Detection Service. Materials and Methods: a cross-sectional study carried out at the Mexican Social Security Institute of San Luis Potosí, Mexico, from august 2012 to september 2013. The universe of records, application forms and results of cervical cytology performed during january to november of 2012 was 4139 . A stratified probabilistic sampling ( 1115 records) was applied. We used a checklist with 32 items divided into 7 sections. Three indicators were used to analyze the data: accuracy, readability and completeness. An ordinal scale was established with the score obtained: low (0-64), medium (65-128) and high (129-192). Student's t-test was used to compare scores on the quality of the records. Results: readability reached a median level in the quality of the records (80.6\%), in the completeness $59.6 \%$ in the high level and in the precision $40.4 \%$ in the average level. Nurses received better quality of records than cytotechnologists $(p=0.05)$. Conclusion: the quality of the records made by the health personnel is medium. MÉD.UIS. 2017;30(3):59-65.

Keywords: Cervical Cancer. Papanicolaou Test. Quality Assurance Health Care. 
¿Cómo citar este artículo?: Gutiérrez-Enríquez SO, Terán-Figueroa Y, Monreal-Delgado L, Nievade-Jesús R, Gaytán-Hernández D. Calidad de los registros en programa de detección oportuna de cáncer cervicouterino. MÉD.UIS. 2017;30(3):59-65.

\section{INTRODUCCIÓN}

El Cáncer Cervicouterino (CC) es la cuarta causa de morbilidad y mortalidad por cáncer entre las mujeres a nivel mundial, en el año 2012 hubo 5'280 000 nuevos casos'. Afecta aproximadamente a 6 de cada 100000 mujeres y representa 275000 muertes al año en los países en desarrollo, lo que corresponde a $88 \%$ de los casos en todo el mundo². Las tasas de mortalidad son tres veces más altas en América Latina y el Caribe que en Norteamérica, evidenciando enormes desigualdades en salud³. En 2009 se reportó una tasa de mortalidad en México de 9,1 por 10000 mujeres y en el estado de San Luis Potosí estuvo en 10,14, es decir, por arriba de la media nacional. En el año 2012 se reportaron para México tasas de incidencia y mortalidad ajustadas por edad de 28,1 y 9,1 por 100000 mujeres respectivamente 5 . Esto podría atribuirse parcialmente a que el cribado se hace de manera general utilizando la Prueba de Papanicolaou (PAP). En los países de altos ingresos se han integrado los servicios de tamizaje basados en la prueba citológica tanto en los servicios médicos como en los de salud pública, con amplia cobertura nacional, lo que ha permitido reducir la incidencia y la mortalidad en las últimas décadas ${ }^{6,7}$. Sin embargo, en los países en desarrollo el éxito no es igual debido a la complejidad de los elementos requeridos.

Desde 1974 se implementa en México el Programa Nacional de Detección Oportuna de Cáncer con el PAP, una prueba que ayuda en la detección oportuna de CC si se realiza adecuadamente ${ }^{8}$, sin embargo, se estima que este programa previene menos del $13 \%$ de los casos de CC en este país, por lo que deberían establecerse mecanismos de capacitación y acreditación del personal encargado de la toma de la muestra, del registro de los datos en la solicitud, así como en la interpretación diagnóstica (enfermeros, citotecnólogos y patólogos), lo anterior aunado a un sistema de control de calidad de los procedimientos en las instituciones públicas y privadas.

En San Luis Potosí, México, uno de los problemas es la deficiencia en la calidad de los registros médicos, según lo reportado por Gutiérrez y cols. ${ }^{10}$, el personal no registró los datos completos de identificación de las usuarias de acuerdo a lo estipulado por el manual de procedimientos de la Secretaria de Salud. En este trabajo se concluye que el contar con módulos específicos facilita el procedimiento, disminuye tiempos de espera, aumenta la privacidad de las pacientes, agiliza el servicio y mejora el control de los registros médicos así como el envío de las muestras y las referencias para la entrega de los resultados.

El propósito de este estudio fue evaluar la calidad de los registros realizados por el personal de salud en el servicio de detección de CC con base en los criterios: legibilidad (que los datos puedan ser leídos con facilidad), completitud (que estén presentes todos los datos que se requieren en la hoja de solicitud y resultados del PAP) y precisión (que se conteste específicamente lo que se solicita), datos de gran interés para la lectura de las muestras, ya que la calidad de los registros permite hacer una lectura más confiable y por consiguiente emitir un diagnóstico más rápido y exacto que beneficiará a la usuaria.

\section{Materiales y métodos}

\section{TIPO DE ESTUDIO, DISEÑO Y MUESTRA}

Estudio transversal realizado en el servicio de Detección Oportuna de Cáncer Cervicouterino de la Unidad de Medicina Familiar (UMF) No. 45 del Instituto Mexicano del Seguro Social de San Luis Potosí, México. El universo de estudio quedó constituido por 4139 registros de resultados de citologías cervicales tomadas a usuarias del programa en el periodo de enero a noviembre de 2012. Dichos registros constituyen el total de las solicitudes de citología cervical de los consultorios de los turnos matutino y vespertino que brindan el servicio.

Para el cálculo del tamaño de la muestra se utilizó la fórmula para la estimación de proporciones de una población finita en el periodo de estudio, con un error estándar del 3\%, un nivel de confianza del $97 \%$ y una proporción esperada del $77,75 \%$. El tamaño de muestra quedó integrado por 743 registros, por el efecto de estratificación se multiplicó por 1,5 quedando un tamaño final de muestra de 1115. 
La estratificación se realizó de acuerdo a la parte proporcional del total de los registros con relación a los turnos matutino (554) y vespertino (561).

Se incluyeron todos los registros realizados por el personal de salud en el periodo estudiado, se excluyeron los que no correspondían al periodo y no se eliminó ninguno. En la institución de salud objeto de estudio, la hoja de solicitud y resultado de la citología cervical es llenada por profesionales en enfermeria al momento de la toma y por citotecnólogos y patólogos en la interpretación de la misma.

\section{INSTRUMENTO DE RECOLECCIÓN DE DATOS}

Para la recolección de datos, se utilizó una lista de verificación elaborada ex-profeso con base en el contenido de la solicitud y reporte de resultados de la citología cervical que se utiliza en la institución, esta tiene un total de 32 ítems. Se dio una ponderación global de 64 puntos por cada dimensión evaluada: legibilidad, completitud y precisión. Se realizó una codificación para el llenado de la lista de verificación de la siguiente manera: 0 = no realizó el registro, 1 = realizó el registro parcialmente, 2 = realizó el registro correctamente. Para medir el nivel de calidad general se utilizó una escala ordinal como sigue: nivel alto: 129 a 192 puntos, medio 65 a 128 puntos y bajo o a 64 puntos. Para comparar la calidad de los registros entre el personal de salud se utilizaron los puntajes obtenidos por grupo (enfermeros y citotecnólogos). Se realizó el mismo procedimiento para los turnos matutino y vespertino.

Para verificar el llenado correcto de la solicitud y resultado de la citología cervical se tomaron en cuenta las instrucciones generales que indica el instructivo para el llenado correcto de dicha solicitud, el cual tiene siete apartados ${ }^{11}$.

- $\quad$ El primero contiene los datos referentes a la identificación de la unidad: unidad médica, delegación y jurisdicción.

- En el segundo apartado se presentan datos de la identificación de la paciente: número de afiliación, número de consultorio, turno, fecha, nombre de la solicitante, edad, lugar de residencia, otro domicilio o referencia de contacto.

- En el tercero se refiere a los antecedentes y las condiciones gineco-obstétricas, así como a los datos de detección de la paciente, los cuales incluyen: fecha de su última citología, condiciones gineco-obstétricas (último parto, tratamiento hormonal, Dispositivo Intrauterino, otro tratamiento ginecológico, posmenopausia, histerectomía y fecha de última menstruación), signos que presenta actualmente en el interrogatorio (flujo, prurito o ardor vulvar, sangrado anormal o ninguno), observaciones a la exploración (cuello aparentemente sano, cuello anormal sin tumoración, cuello anormal con tumoración, no se observa cuello) y si la paciente fue derivada con el médico familiar

- El cuarto apartado describe el utensilio con el que se tomó la muestra: espátula de Ayre, citobrush, pipeta, abatelenguas y otro.

- En el quinto apartado se escriben los datos del tomador de la muestra citológica (nombre y matrícula del personal de salud).

- $\quad$ El sexto se refiere a: 1) Los resultados de la citología cervical (laboratorio en donde se analizó la muestra, número citológico, fecha de recepción delamuestra,fechadelainterpretación de la muestra, características de la muestra (presencia de células endocervicales, metaplasia epidermoide o inadecuada para el diagnóstico)); 2) Diagnóstico citológico (negativo a cáncer, negativo con proceso inflamatorio, NIC I, NIC II, NIC III; NIC III "in situ”, microinvasor, invasor, adenocarcinoma, maligno no especificado); 3) Hallazgos adicionales (imagen de virus de papiloma humano, imagen de virus del herpes, tricomonas, bacterias, hongos, otros, ninguno); 4) Especificación de repetición del estudio y el motivo (células cervicales ausentes, muestra mal fijada, muestra mal teñida, exudado inflamatorio abundante, sangrado, laminilla rota o extraviada, muestra contaminada, otra).

- El séptimo apartado contiene los datos: nombre y matrícula del citotecnólogo que examinó la muestra. Si la muestra fue revisada por el patólogo: nombre, firma y matrícula de este.

\section{ANÁLISIS ESTADÍSTICO}

La información fue analizada en el programa SPSS versión 18. Se realizó un análisis descriptivo en porcentajes. Se aplicó la prueba $t$ de student para comparar los puntajes obtenidos a partir 
de la calidad de los registros, utilizando el $95 \%$ de confianza con un valor a del $5 \%$. En esta prueba la hipótesis nula es que las medias de los puntajes son iguales, para comparar las medias entre los grupos (enfermería y citotecnólogos), así mismo, para realizar esta comparación entre los turnos (matutino y vespertino).

\section{Resultados}

En la calidad general de los registros predominó el nivel medio con un $89 \%$ (Ver Gráfica 1). En los registros por criterio, se observó que en la legibilidad la mayoría posee calidad media con un porcentaje del $80.6 \%$. En el criterio de completitud, se encontró una calidad alta en el 59.6\%. En el criterio de precisión se encontró que un $40.4 \%$ posee una calidad media (Ver Gráfica 2).

En el nivel de calidad global por apartados del contenido de las solicitudes se encontró que, el primer, segundo y quinto apartado poseen calidad media (100, 67.9, y 55.7\% respectivamente). En el tercero, cuarto y sexto apartado hay calidad alta (57,1, 89,9 y 53,5\% respectivamente), mientras que en el apartado séptimo preponderó la calidad baja con un $98,4 \%$ (Ver Tabla 1).

En cuanto a la calidad de los registros realizados por el personal de salud, el profesional de enfermería los hace con una calidad media en un $51,7 \%$ y alta en un 48,3\%. El citotecnólogo registra con calidad media en un $95 \%$ y alta en un 0,9\%. En relación al puntaje obtenido por el personal de salud de acuerdo a la disciplina, enfermería obtuvo una media de 71.4 puntos en una escala de $0-108$, por otro lado, los citotecnólogos obtuvieron una media de 40,6 puntos en una escala de 0-84 (Ver Tabla 2). Así, el personal de Enfermería obtuvo una mejor calificación en la calidad de los registros que los citotecnólogos ya que la diferencia de los puntajes obtenida por cada uno de estos grupos fue significativamente mayor en este con una $p$ menor de 0.05 .

En relación al turno, el matutino obtuvo el mayor número con 113,4 como media, comparada con el vespertino el cual fue de 110,6. La diferencia de los puntajes obtenida por cada uno de estos turnos fue significativamente mayor para el matutino con una $p$ menor de 0,05 (Ver Tabla 3).

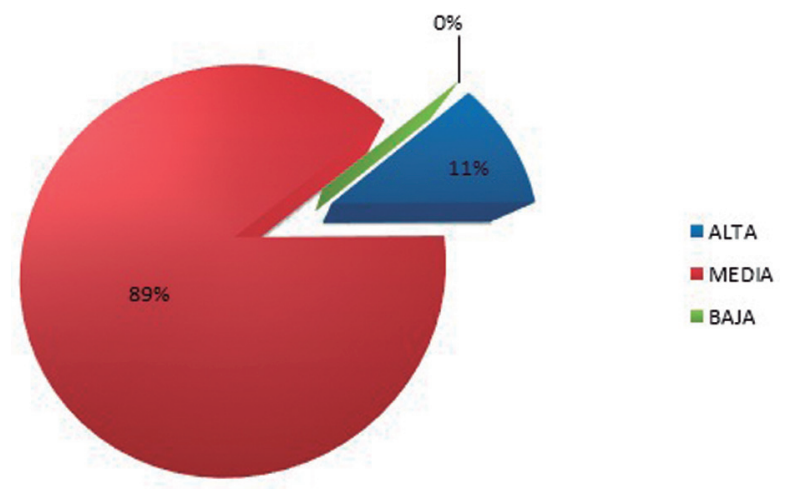

Gráfica 1. Nivel de calidad global de los registros realizados por el personal de salud en el programa de DOC. $(n=1115)$

Fuente: Autores.

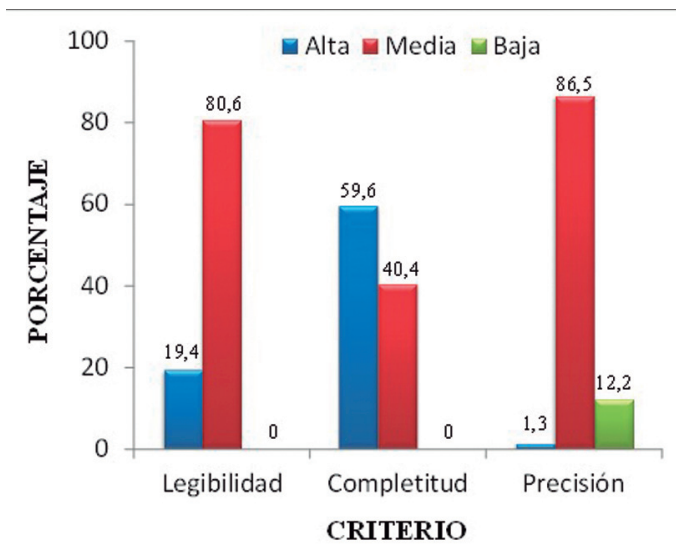

Gráfica 2. Nivel de calidad por criterio de evaluación de los registros realizados por el personal de salud en el programa DOC. $(n=1115)$ Fuente: Autores.

\section{Discusión}

Las tendencias del mundo actual en el campo de la salud suponen nuevos desafíos para el profesional en el logro de la calidad, lo cual implica el desarrollo de instrumentos que evalúen la calidad con la finalidad de garantizar y optimizar los servicios prestados en el área $^{12}$. Una de estas herramientas es el registro en la solicitud de la toma de la citología cervical, elemento principal que sirve en comunicación entre el personal que toma la muestra, el laboratorio, el citotecnólogo y la paciente. Los datos de identificación de la usuaria y la información clínica completa son indispensables para una adecuada correlación e interpretación de los hallazgos cito-morfológicos. Un registro completo, legible y preciso ayudará a obtener un diagnóstico certero y puntual ${ }^{13}$. 
En este estudio se identificó que de manera general el personal de enfermería y los citotecnólogos tienen un desempeño de nivel medio en el registro del formato de solicitud y resultados de las citologías cervicales, esto tiene relación con lo que refiere Grespan Setz y cols. en 2009 en su estudio hecho en Brasil sobre la evaluación de la calidad de los registros de enfermería a través de la auditoría, en donde los proveedores del servicio realizaron sus registros de manera regular mostrando fallas importantes en la legibilidad y en la precisión ${ }^{14}$.

Tabla 1. Nivel de calidad global por apartados de los registros de las solicitudes y resultados de las citologías cervicales realizados por el personal de salud

\begin{tabular}{|c|c|c|c|c|c|c|}
\hline \multicolumn{7}{|c|}{ NIVEL DE CALIDAD } \\
\hline \multirow{2}{*}{$\begin{array}{c}\text { Apartados de la } \\
\text { solicitud }\end{array}$} & \multicolumn{2}{|c|}{ Alta } & \multicolumn{2}{|c|}{ Media } & \multicolumn{2}{|c|}{ Baja } \\
\hline & $n$ & $\% *$ & $n$ & $\% *$ & $\mathbf{n}$ & $\% *$ \\
\hline $\begin{array}{l}\text { Identificación de la } \\
\text { unidad }\end{array}$ & 0 & 0 & 1115 & 100,0 & 0 & 0 \\
\hline $\begin{array}{c}\text { Identificación de la } \\
\text { solicitante }\end{array}$ & 355 & 31,8 & 757 & 67,9 & 3 & 0,3 \\
\hline $\begin{array}{c}\text { Antecedentes } \\
\text { gineco-obstétricos }\end{array}$ & 637 & 57,1 & 330 & 29,6 & 148 & 13,3 \\
\hline $\begin{array}{l}\text { Utensilio con que } \\
\text { se tomó la muestra }\end{array}$ & 1002 & 89,9 & 73 & 6,5 & 40 & 3,6 \\
\hline $\begin{array}{l}\text { Datos del personal } \\
\text { de salud que tomó } \\
\text { la muestra }\end{array}$ & 381 & 34,2 & 621 & 55,7 & 113 & 10,1 \\
\hline $\begin{array}{l}\text { Datos de los } \\
\text { resultados de la } \\
\text { citología cervical }\end{array}$ & 596 & 53,5 & 514 & 46,1 & 5 & 0,4 \\
\hline $\begin{array}{l}\text { Datos del personal } \\
\text { de salud que } \\
\text { interpreto la } \\
\text { muestra }\end{array}$ & 10 & 0,9 & 8 & 0,7 & 1097 & 98,4 \\
\hline
\end{tabular}

* Sobre 1115 registros

Fuente: Autores.

Tabla 2. Puntaje general de registros que realizó el personal de salud en las solicitudes y resultados de las citologías cervicales.

\begin{tabular}{|c|c|c|c|c|c|}
\hline $\begin{array}{c}\text { Personal de } \\
\text { salud }\end{array}$ & Escala & Mínimo & Máximo & D.E. & Media \\
\hline Enfermería & $0-108$ & 40 & 96 & 11 & 71.4 \\
\hline Citotecnólogo & $0-84$ & 10 & 70 & 6.4 & 40.6 \\
\hline
\end{tabular}

$\mathrm{t}=8390 p=<0,001$

D.E. = Desviación Estándar

Fuente: Autores.

Así mismo, Villeda García y cols. en 2010, encontraron que en el Instituto Nacional de Salud de la Ciudad de México la calidad de 122 registros de expedientes era regular, entre las principales deficiencias se encontraban las omisiones del nombre y firma de quien los elabora, así como de la vía de administración de medicamentos; adicionalmente se identificó ilegibilidad y tachaduras en los documentos, lo cual repercute directamente en la calidad de la atención y en la seguridad del paciente ${ }^{15}$.

Tabla 3. Puntaje obtenido en los registros realizados por el personal de salud de solicitudes y resultados de las citologías cervicales por turno

\begin{tabular}{|c|c|c|c|c|}
\hline Turno & Mínimo & Máximo & Media & D.E. \\
\hline Matutino & 70 & 151 & 113,4 & 113,9 \\
\hline Vespertino & 77 & 144 & 110,6 & 110,6 \\
\hline
\end{tabular}

Escala de medición general de 0 - 192 puntos $p=0,001 ;$ D. E. = Desviación estándar Fuente: Autores.

En el presente estudio las principales debilidades encontradas fueron la escritura ilegible, el no uso de tinta negra como está señalado en el manual de procedimientos y el empleo de iniciales o palabras cuyo significado es inentendible. Este aspecto es de vital importancia ya que el personal que interpreta las muestras puede tener dificultades en emitir un diagnóstico a partir de la falta de información relevante. En lo que se refiere a la completitud, la mitad del personal obtuvo un nivel de calidad medio. Se identificó que donde existen más fallas es en los registros de los antecedentes, así como en la identificación de los pacientes y en la sección en donde se reportan los resultados de las muestras.

En el apartado de precisión la mayoría del personal obtuvo un nivel de calidad medio, se identificaron problemas de omisión de datos, ubicación de la información fuera del espacio asignado y en el orden de los mismos; resultado similar al encontrado en un estudio sobre la calidad de los registros de las historias clínicas realizado por Pablo-Rocano en 2008 en el servicio de emergencias de un Hospital General, en donde se observó que todos los registros fueron auditados y calificados como regulares debido a la falta de los datos de filiación, estos registros estuvieron incompletos en un $70 \%$ y no se enfatizó el motivo de la consulta ${ }^{16}$. Por otra parte, en el 2012 Paolino y Arrossi, en un estudio realizado en Argentina en 165 mujeres, analizaron el motivo de abandono del proceso y seguimiento del tratamiento de las lesiones precursoras del cáncer de cuello. Los resultados indican que no fue posible contactar a 79 pacientes debido a que sus direcciones estaban incompletas, eran erróneas o inexistentes ${ }^{17}$.

En el nivel de calidad del registro por sección, de acuerdo a lo solicitado en la hoja de solicitud y 
resultados de la citología cervical, se observó que los apartados en donde se presentan más problemas son la identificación de la unidad, identificación de la solicitante y nombre del personal que tomó la muestra, de manera general no se anota el segundo domicilio para localizar a la paciente además la dirección y edad no son legibles.

Otro aspecto importante de analizar es la estructura de los formatos utilizados para los registros. Se encontró que no existe un espacio para anotar las consideraciones microscópicas del espécimen por los citotecnólogos (como el saber si la muestra contiene células endocervicales, células de la zona de transformación o si cumple las especificaciones del sistema Bethesda 2001 para la citología cervical) ${ }^{18}$, dato importante ya que a partir de él se toma la decisión de repetir o no la muestra. Este problema ya ha sido encontrado y documentado en otros estudios $^{19}$ en donde se analiza la estructura de los formatos, ninguno tiene el espacio para anotar dichas implicaciones microscópicas.

Con relación a los datos registrados por el personal de salud responsable de interpretar las muestras se observó una calidad baja, sobre todo en la precisión y legibilidad, se observó la ausencia en su mayoría de la firma del citotecnólogo, solo colocaron su nombre utilizando un sello, además no reportan los hallazgos adicionales que es una sección que viene en la hoja de citología. En muy pocas solicitudes está registrada la revisión por el patólogo. La Norma Oficial Mexicana NOM-014-SSA2-1994, para la prevención, detección, diagnóstico, tratamiento, control y vigilancia epidemiológica del cáncer cervicouterino, refiere que el $10 \%$ de las muestras con resultado negativo y el $100 \%$ con resultado positivo deben revisarse por un patólogo ${ }^{20}$, esto es fundamental porque es el momento en que se pueden identificar casos de falsos positivos o falsos negativos, situación que en nuestro medio es factible de presentarse como lo reporta Gutiérrez y cols. en 2016 en un estudio sobre la concordancia diagnóstica en la interpretación de las citologías cervicales en una institución de salud pública de San Luis Potosí, México, en donde los citotecnólogos dieron lectura a muestras de citologías cervical con diagnósticos variados previamente interpretadas por un patólogo. Se encontró un alto porcentaje de diagnósticos que no coincidieron con los emitidos por el patólogo ${ }^{21}$.

En el estudio la calidad de los registros por turnos se calificó como media. Se encontró una relación entre la calidad de los registros que realiza el personal de ambos turnos a favor del turno matutino, sin embargo, dichas diferencias se deben analizar con más cuidado a fin de establecer las causas exactas ya que este fenómeno varía de acuerdo al contexto estudiado, lo cual se pudo observar en el trabajo realizado por Villeda García y cols. en 2010 en donde el turno con mayor incumplimiento en los registros era el matutino ${ }^{15}$. Por otro lado, se reportó una diferencia estadísticamente significativa entre el personal de enfermería y los citotecnólogos, donde los primeros realizan con mayor calidad los registros, lo cual coincide con un estudio realizado por Rodríguez Almada y cols. en 2009 en un estudio llevado a cabo en Uruguay, en donde detectaron notorias inconsistencias en la información registrada por el personal médico ${ }^{22}$.

Algunas limitantes encontradas para realizar este estudio fueron las siguientes: dificultad en localizar los datos a evaluar, ya que no todos se encontraban en el mismo lugar, además, existe un retraso importante en el proceso de registro, captura, procesamiento y entrega de la información ya que todo se realiza de manera manual. Para futuras investigaciones se recomienda analizar variables como la capacitación y el apego a las normas técnicas en cuanto a los registros en otras áreas operativas que tengan relación con el programa de Detección Oportuna de Cáncer Cervicouterino. Por otra parte, es deseable que se realicen reformas estructurales en los sistemas de información en las instituciones de salud, de manera que los registros manuales se realicen de manera electrónica, lo que sin duda redundará en una mejor organización y utilización de los datos obtenidos.

\section{Conclusiones}

La calidad de los registros fue media; la precisión obtuvo el nivel más bajo, seguido por la completitud y la legibilidad; los citotecnólogos obtuvieron la escala más baja de calidad. Es necesario implementar intervenciones dirigidas a la capacitación del personal de salud, ya que el apego a la normatividad institucional en lo que se refiere a los registros no es la esperada. Los proveedores de servicio son los encargados de ejecutar el programa de Detección Oportuna de Cáncer y su desempeño tendrá una relación directa con la calidad brindada al paciente por lo que los tomadores de decisiones o administradores tendrán que orientar sus esfuerzos a la planeación de sistemas más efectivos e innovadores. 


\section{FinANCIACIÓN}

El estudio fue autofinanciado por los autores.

\section{Consideraciones ÉtICAS}

El protocolo contó con la autorización del Comité de Ética e Investigación de la Facultad de Enfermería y Nutrición de la UASLP (registro CEIFE-2013-046) así como del Comité Local de Investigación y Ética en Investigación en Salud 2402 del Hospital General de Zona-MF-No. 1 de San Luis Potosí, México (registro R-2013-2402-47).

\section{CONFLICTO DE INTERESES}

Los autores manifiestan que no hubo conflicto de intereses en la ejecución de la investigación.

\section{RefERENCIAS BibLIOGRÁFICAS}

1. Ferlay J, Soerjomataram I, Dikshit R, Eser, S, Mathers, C, Rebelo, et al. Cancer incidence and mortality worldwide: Sources, methods and major patterns in GLOBOCAN 2012. Int J Cancer. 2015;136(5):e359-86.

2. Siegel RL, Miller KD, Jemal A. Cancer statistics, 2015. CA Cancer J Clin [Internet]. 2015 [Citado 2017 Sep 09];65(1):5-29. Disponible en: https://www.ncbi.nlm.nih.gov/pubmed/25559415

3. Pan American Health Organization. [Internet]. Pan American Health Organization; Cervical Cancer; 2016 Sep 2. [Cited 2016 Oct 27]. Available from: http://www.paho.org/hq/ index.php?option $=$ com content \&view $=$ article\&id $=5420$ $\% 3$ A2011-cervical-cancer\&catid $=3595 \% 3$ Acervical-cancer content 2 \&Itemid $=3637$ \&lang $=\mathrm{es}$

4. Instituto Nacional de Estadística y Geografía. [Internet]. Gobierno Federal de México; 2012. [Citado 2017 Sep 09]. Disponible en: http://www.inegi.org.mx/

5. Word Health Organization. Globocan 2012: Estimated Cancer Incidence, Mortality and Prevalence Worldwide in 2012 [Internet]. IARC, WHO. [Citado 2016 Feb 27]. Disponible en: http://globocan.iarc.fr/Pages/fact_sheets_population:aspx

6. Sahasrabuddhe VV, Parham GP, Mwanahamuntu MH, Vermund SH. Cervical Cancer Prevention in Low-and MiddleIncome Countries: Feasible, Affordable, Essential. Cancer Res. [Internet]. 2012 [Citado 2017 Sep 09]; 5(1): 12-17. Disponible en: 10.1158/1940-6207.CAPR-11-0540

7. Forouzanfar MH, Foreman KJ, Delossantos AM, Lozano R, Lopez AD, Murray CJ, et al. Breast and Cervical Cancer in 187 Countries Between 1980 and 2010: A Systematic Analysis. Lancet. [Internet]. 2011 [Citado 2017 Sep 09]; 378(11):14611484. Disponible en: 10.1016/S0140-6736(11)61351-2

8. Hidalgo Martínez AC. Cervical cancer, its Impact in Mexico and why does not work the National Program of Early Detection. Rev
Biomed. 2006; 17 (1): 81-84.

9. Secretaría de Salud. Gobierno de México. Dirección general de calidad y educación para la salud; 2007. [Internet]. DGCES. [Citado 2016 Feb 19]. Disponible en: http://portal.salud.gob. $\mathrm{mx} /$ sites/salud/descargas/pdf/evaluaciones/ssas/sicalidad/pi sicalidad.pdf

10. Gutiérrez-Enríquez SG, Gaytán-Hernández D, Zamarripa-Leyva JM, Terán-Figueroa. Desempeño del personal de salud en la toma de las citologías cervicales: conocimientos teóricos y ejecución práctica. Ginecol Obstet Mex. 2014;82(5):296-306.

11. Secretaría de Gobierno de México. Norma Oficial Mexicana NOM-004-SSA3-2012, Del expediente clínico; Oct 2012 [Internet]. Secretaría de Salud. [Citado 2016 Sep 27]. Disponible en: http://dof.gob.mx/nota_detalle_popup. php?codigo $=5272787$

12. Torres Santiago M, Zárate Grajales RA, Matus Miranda R. Calidad de los registros clínicos de enfermería: Elaboración de un instrumento para su evaluación. Revista Enfermería Universitaria ENEO-UNAM. 2011;8(1):17-25.

13. Díaz Cuspoca MC, Parra Saad EA. Guía control de calidad para la toma, procesamiento e interpretación en muestras de citología de cuello uterino [Internet]. Segunda edición. Bogotá, Colombia: Instituto Nacional de Salud. Ministerio de la protección social; 2009 [Citado 2016 Ago 20]. Disponible en: https://issuu.com/ institutonacionaldesalud/docs/gu_a_control_de_calidad_en_ muestras de citolog a

14. Grespan V, D'Innocenzo M. Evaluation of the quality of nursing documentation though the review of patient medical records. Acta paul. enferm. 2009;22(3):313-7. http://dx.doi.org/10.1590/ S0103-21002009000300012.

15. Villeda FL, Córdoba MA, Rodríguez J, Balbuena S, Hernández M, Díaz ML, et al. Level of compliance with the Mexican Official Standard 168-SSA1-1998 on clinical records by the nursing staff of a National Institute of Health. Revista CONAMED [internet] 2010 [citado Sep 28 2016];15(4). Disponible en: http://www.dgdiconamed.salud.gob.mx/ojs-conamed/index.php/revconamed/rt/ printedFriendly/260/472

16. Pablo E. Quality evaluation of the records of the dead patient's medical charts in the Emergency Department of a General Hospital. Rev Soc Peru Med Interna. 2008; 21(2):51-4.

17. Paolino M, Arrossi S. Analysis of the reasons for abandoning the follow-up and treatment process in women with pre-cancerous cervical lesions in the province of Jujuy: implications for health management. Salud Colect. 2012;8(3):247-61.

18. Solomon D, Davey D, Kurman R, Moriarty A, O'Connor D, Prey M, et al. The 2001 Bethesda System: terminology for reporting results of cervical cytology. JAMA, 2002;287(16):2114-9.

19. Gutiérrez S. Calidad de la toma de citologías cervicales y factores asociados en el personal de salud de la secretaria de salud de San Luis Potosí. [tesis doctoral], San Luis Potosí; jurisdicción 1, SSA; 2006.

20. Secretaría de Salud. Norma Oficial Mexicana NOM-014SSA2-1994, Para la prevención, detección, diagnóstico, tratamiento, control y vigilancia epidemiológica del cancer cervico uterino. Estados Unidos Mexicanos. 2007.

21. Gutiérrez SO, Chávez L, Teran Y, Gaytán D, Oros C, Gallegos V, et al. Concordance in the Interpretation of Cervical Cytology for the Early Diagnosis of Cervical Cancer. Open J Obstet Gynecol. 2016;6(12):714-24.

22. Rodríguez H, Ciriacos C, Piñeyrúa M, Logaldo R, González D. Quality registration of death certificate in a public referral hospital. Montevideo, Uruguay, october-november 2009. Rev Med Urug. 2010;26(4):216-23. 